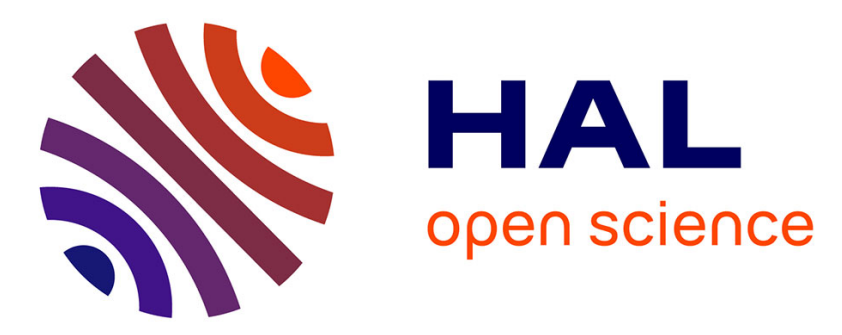

\title{
Circularly polarized few-optical-cycle solitons in the short-wave-approximation regime
}

Hervé Leblond, Houria Triki, Dumitru Mihalache

\section{To cite this version:}

Hervé Leblond, Houria Triki, Dumitru Mihalache. Circularly polarized few-optical-cycle solitons in the short-wave-approximation regime. Physical Review A: Atomic, molecular, and optical physics [1990-2015], 2011, 84 (2), pp.023833. 10.1103/PhysRevA.84.023833 . hal-03187682

\section{HAL Id: hal-03187682 \\ https://univ-angers.hal.science/hal-03187682}

Submitted on 1 Apr 2021

HAL is a multi-disciplinary open access archive for the deposit and dissemination of scientific research documents, whether they are published or not. The documents may come from teaching and research institutions in France or abroad, or from public or private research centers.
L'archive ouverte pluridisciplinaire HAL, est destinée au dépôt et à la diffusion de documents scientifiques de niveau recherche, publiés ou non, émanant des établissements d'enseignement et de recherche français ou étrangers, des laboratoires publics ou privés. 


\title{
Circularly polarized few-optical-cycle solitons in the short-wave-approximation regime
}

\author{
Hervé Leblond, ${ }^{1}$ Houria Triki, ${ }^{2}$ and Dumitru Mihalache ${ }^{1,3,4}$ \\ ${ }^{1}$ Laboratoire de Photonique d'Angers, EA 4464, Université d'Angers, 2 Boulevard Lavoisier, F-49045 Angers Cedex 01, France \\ ${ }^{2}$ Radiation Physics Laboratory, Department of Physics, Faculty of Sciences, Badji Mokhtar University, Post Office Box 12, \\ 23000 Annaba, Algeria \\ ${ }^{3}$ Horia Hulubei National Institute for Physics and Nuclear Engineering (IFIN-HH), 407 Atomistilor, \\ RO-077125 Magurele-Bucharest, Romania \\ ${ }^{4}$ Academy of Romanian Scientists, 54 Splaiul Independentei, RO-050094 Bucharest, Romania
}

(Received 21 April 2011; published 18 August 2011)

\begin{abstract}
We consider the propagation of few-cycle pulses (FCPs) beyond the slowly varying envelope approximation in media in which the dynamics of constituent atoms is described by a two-level Hamiltonian by taking into account the wave polarization. We consider the short-wave approximation, assuming that the resonance frequency of the two-level atoms is well below the inverse of the characteristic duration of the optical pulse. By using the reductive perturbation method (multiscale analysis), we derive from the Maxwell-Bloch-Heisenberg equations the governing evolution equations for the two polarization components of the electric field in the first order of the perturbation approach. We show that propagation of circularly polarized (CP) few-optical-cycle solitons is described by a system of coupled nonlinear equations, which reduces in the scalar case to the standard sine Gordon equation describing the dynamics of linearly polarized FCPs in the short-wave-approximation regime. By direct numerical simulations, we calculate the lifetime of CP FCPs, and we study the transition to two orthogonally polarized single-humped pulses as a generic route of their instability.
\end{abstract}

DOI: 10.1103/PhysRevA.84.023833

PACS number(s): 42.65.Tg, 42.65.Re, 05.45.Yv

\section{INTRODUCTION}

Ultrashort optical pulses with duration of merely a few femtoseconds find diverse applications in the area of light-matter interactions, high-order harmonic generation, extreme [1] and single-cycle [2] nonlinear optics, and attosecond physics [3]; for a review of earlier works in this area see Ref. [4]. Recent experimental advances in the study of the wave dynamics of few-cycle pulses (FCPs) in nonlinear optical media have paved the way for the development of new theoretical approaches to modeling their propagation in realistic physical systems. We mention here only a recent work demonstrating the synthesis of a single cycle of light by using compact erbium-doped fiber technology [5]; the obtained pulse duration of only $4.3 \mathrm{fs}$ was close to the shortest possible value for a data bit of information transmitted in the near-infrared spectrum of light, at a wavelength of $1300 \mathrm{~nm}$.

Three classes of main dynamical models for FCPs have been investigated in detail in the past: (i) the quantum approach [6-9], (ii) refinements within the framework of the slowly varying envelope approximation (SVEA) of the nonlinear Schrödinger-type envelope equations [10-13], and (iii) non-SVEA models [14-21]. Recent works on FCPs deal with few-cycle light bullets created by femtosecond filaments [22], ultrashort spatiotemporal optical solitons in quadratic nonlinear media [23], ultrashort spatiotemporal optical pulse propagation in cubic (Kerr-like) media without the use of the SVEA [24], single-cycle gap solitons generated in resonant two-level dense media with a subwavelength structure [25], few-cycle propagating surface plasmon polariton wave packets [26], and the possibility of generating few-cycle dissipative optical solitons [27,28]. We also mention recent studies of ultrafast pulse propaga- tion in a mode-locked laser cavity in the few-femtosecond pulse regime and the derivation of a master mode-locking equation for ultrashort pulses [29], the experimental study of intrinsic chirp of single-cycle pulses [30], and the proposed method of generating extremely short unipolar half-cycle pulses based on resonant propagation of a few-cycle pulse through asymmetrical media with periodic subwavelength structure [31].

It has been proved in recent years that the propagation of FCPs in Kerr media can be described beyond the SVEA by using the modified Korteweg-de Vries (mKdV) [15], sine Gordon (sG) $[16,17]$, or $\mathrm{mKdV}-\mathrm{sG}$ equations [18,19]. It is well known that the $\mathrm{mKdV}$ and $\mathrm{sG}$ equations are completely integrable by means of the inverse scattering transform (IST) method [32,33], whereas the $\mathrm{mKdV}$-sG equation is completely integrable only if some condition between its coefficients is satisfied [34].

The aim of this work is to study the propagation of circularly polarized (CP) few-optical-cycle pulses in optical media beyond SVEA and when the frequency of the transition is far below the characteristic wave frequency (the so-called short-wave-approximation regime). Note that the characteristic pulse duration is still assumed to be about 1 fs, corresponding to an optical pulse of only a few cycles. We therefore focus on the vectorial character of the electric field by taking into account the wave polarization effects and by applying the reductive perturbation method (multiscale analysis) to the Maxwell-Bloch equations. Notice that other vectorial non-SVEA models have been also proposed $[35,36]$; however, they were not carefully justified from physical point of view but built from only an analogy with common SVEA models. However, recently [37] we found $\mathrm{CP}$ few-optical-cycle solitons in cubic nonlinear media in the 
long-wave-approximation regime and beyond the SVEA. Assuming that the frequency of the transition is far above the characteristic wave frequency (i.e., in the long-waveapproximation regime), we showed that propagation of FCPs, taking into account the wave polarization, is adequately described by the nonintegrable complex mKdV equation. We have also concluded that the CP FCP soliton becomes unstable when the angular frequency is less than 1.5 times the inverse of the pulse duration. As a result, the unstable subcycle pulses decay into linearly polarized half-cycle pulses, whose polarization direction slowly rotates around the propagation axis [37].

In the present work we show, by using the reductive perturbation method (multiscale analysis) at its first-order limit, that the evolution of the two wave amplitudes describing the vectorial character of ultrashort optical pulse propagation in a system of two-level atoms is adequately described by a system of coupled nonlinear equations, which reduces to the common sine Gordon equation in the scalar case (i.e., the case of linearly polarized few-cycle solitons).

Circularly polarized short-pulse propagation in a system of two-level atoms has already been studied in the frame of selfinduced transparency [38]. The existence of localized solutions of Maxwell-Bloch-type systems beyond the SVEA has been considered $[39,40]$. However, not all the coupling mechanisms between the polarization components were taken into account. The authors of Ref. [40] took an essential coupling term into account through the out-of-phase polarization, which allowed them to show that the pulse solution valid within the SVEA could not be generalized beyond it by means of corrections terms.

The paper is organized as follows. After introduction of the corresponding fast and slow variables, we derive the dynamical equations governing the propagation of vector femtosecond optical solitons (circularly polarized few-opticalcycle solitons) in the short-wave approximation by developing a multiple scaling approach to the Maxwell-Bloch-Heisenberg equation up to the first order in the small parameter $\varepsilon$. In Sec. III, we study in detail the mathematical properties of the derived coupled system of nonlinear equations, and we get the corresponding conservation laws. We also obtain linearly polarized few-cycle solitons as a limiting case of the more general vectorial model. In Sec. IV, we perform a comprehensive study of the circularly polarized few-optical-cycle solitons in the short-wave-approximation regime, and we give an approximate analytical expression of the circularly polarized pulse. For the sake of completeness, we also consider the slowly varying envelope approximation derived from the general system of coupled nonlinear equations obtained in Sec. II. As a result, we get the corresponding system of two coupled nonlinear Schrödinger (NLS) equations. We also study the stability of circularly polarized pulses, which can be done analytically within the slowly varying envelope approximation. In Sec. V, we calculate by adequate numerical methods the lifetime of circularly polarized few-cycle pulses, and we study the transition to two orthogonally polarized single-humped pulses as a generic decay scenario of unstable circularly polarized solitons. Finally, in Sec. VI we summarize the results of our analysis and indicate some other possible extensions of this work.

\section{DERIVATION OF THE GOVERNING DYNAMICAL EQUATIONS FOR CIRCULARLY POLARIZED FEW-OPTICAL-CYCLE SOLITONS IN THE SHORT-WAVE APPROXIMATION}

\section{A. Basic equations}

We consider a two-level model, in which the excited state is degenerated twice, corresponding to oscillations along the $x$ and $y$ axes. The free Hamiltonian is thus

$$
H_{0}=\hbar\left(\begin{array}{ccc}
\omega_{a} & 0 & 0 \\
0 & \omega_{b} & 0 \\
0 & 0 & \omega_{b}
\end{array}\right)
$$

and the resonance angular frequency is $\Omega=\omega_{b}-\omega_{a}>0$. The electric field $\vec{E}$ is coupled with the atoms by the Hamiltonian

$$
H=H_{0}-\vec{\mu} \cdot \vec{E},
$$

in which the dipolar momentum operator $\vec{\mu}=\mu_{x} \vec{e}_{x}+\mu_{y} \vec{e}_{y}$ is given by

$$
\mu_{x}=\left(\begin{array}{ccc}
0 & \mu & 0 \\
\mu^{*} & 0 & 0 \\
0 & 0 & 0
\end{array}\right), \quad \mu_{y}=\left(\begin{array}{ccc}
0 & 0 & \mu \\
0 & 0 & 0 \\
\mu^{*} & 0 & 0
\end{array}\right) .
$$

Then the evolution of the atoms is governed by the Heisenberg equation

$$
i \hbar \partial_{t} \rho=[H, \rho],
$$

in which $\rho$ is the density matrix, and the evolution of the electric field $\vec{E}$ is governed by the wave equation

$$
\partial_{z}^{2} \vec{E}=\frac{1}{c^{2}} \partial_{t}^{2}(\vec{E}+4 \pi \vec{P}),
$$

where $c$ is the speed of light in vacuum and the polarization density $\vec{P}$ is given by

$$
\vec{P}=N \operatorname{Tr}(\rho \vec{\mu}) .
$$

\section{B. Short-wave approximation}

The short-wave approximation is performed according to the general theory developed in Refs. [16,41,42]. We denote by $(u, v, 0)$ the components of the electric field $\vec{E}$ in the $(x y z)$ frame, by $(P, Q, 0)$ the ones of $\vec{P}$, and by $\rho_{i j}, i, j=1,2,3$, the elements of the Hermitian matrix $\rho$. All these quantities are expanded in the power series of a small parameter $\varepsilon$ as

$$
\vec{E}=\vec{E}^{0}+\varepsilon \vec{E}^{1}+\varepsilon^{2} \vec{E}^{2}+\cdots
$$

and so on. We introduce fast and slow variables

$$
\tau=\left(t-\frac{z}{V}\right), \quad \zeta=\varepsilon z,
$$

so that

$$
\partial_{t}=\partial_{\tau}, \quad \partial_{z}=-\frac{1}{V} \partial_{\tau}+\varepsilon \partial_{\zeta} .
$$

The expansion (7) and fast and slow variables (8) are reported into the basic equations (5) and (4), and the perturbative scheme is solved order by order. 


\section{Order $\varepsilon^{0}$}

The Heisenberg equation (4) at order $\varepsilon^{0}$ yields

$$
i \hbar \partial_{\tau} \rho^{0}=-\left[\vec{\mu} \cdot \vec{E}_{0}, \rho^{0}\right],
$$

that is,

$$
\begin{gathered}
i \hbar \partial_{\tau} \rho_{11}^{0}=-\left(\mu \rho_{12}^{0 *}-\rho_{12}^{0} \mu^{*}\right) u_{0}-\left(\mu \rho_{13}^{0 *}-\rho_{13}^{0} \mu^{*}\right) v_{0}, \\
i \hbar \partial_{\tau} \rho_{22}^{0}=-\left(\mu^{*} \rho_{12}^{0}-\rho_{12}^{0 *} \mu\right) u_{0} \\
i \hbar \partial_{\tau} \rho_{33}^{0}=-\left(\mu^{*} \rho_{13}^{0}-\rho_{13}^{0 *} \mu\right) v_{0} \\
i \hbar \partial_{\tau} \rho_{12}^{0}=-\mu\left(\rho_{22}^{0}-\rho_{11}^{0}\right) u_{0}-\mu \rho_{23}^{0 *} v_{0} \\
i \hbar \partial_{\tau} \rho_{13}^{0}=-\mu \rho_{23}^{0} u_{0}-\mu\left(\rho_{33}^{0}-\rho_{11}^{0}\right) v_{0} \\
i \hbar \partial_{\tau} \rho_{23}^{0}=-\mu^{*} \rho_{13}^{0} u_{0}+\rho_{12}^{0 *} \mu v_{0}
\end{gathered}
$$

We check that Eqs. (11)-(13) satisfy the normalization condition for the density matrix, that is, $\partial_{\tau} \operatorname{Tr} \rho^{0}=0$.

Assuming that the electric field components $u$ and $v$ vanish as $\tau$ tends to $-\infty$, by integrating (14) and (15) and incorporating them into (11) we get

$$
\begin{aligned}
\partial_{\tau} \rho_{11}^{0}= & \frac{2|\mu|^{2}}{\hbar^{2}}\left[u_{0} \int_{-\infty}^{\tau}\left(w_{1} u_{0}+\sigma v_{0}\right)\right. \\
& \left.+v_{0} \int_{-\infty}^{\tau}\left(\sigma u_{0}+w_{2} v_{0}\right)\right],
\end{aligned}
$$

where we have set the population inversion quantities

$$
w_{1}=\rho_{22}^{0}-\rho_{11}^{0}, \quad w_{2}=\rho_{33}^{0}-\rho_{11}^{0},
$$

and

$$
\sigma=\operatorname{Re} \rho_{23}^{0}=\frac{\rho_{23}^{0}+\rho_{23}^{0 *}}{2} .
$$

Then by incorporating (14) into (12), we get

$$
\partial_{\tau} \rho_{22}^{0}=-\frac{2|\mu|^{2}}{\hbar^{2}} u_{0} \int_{-\infty}^{\tau}\left(w_{1} u_{0}+\sigma v_{0}\right) .
$$

Integrating Eq. (15) and incorporating it into (13) yields

$$
\partial_{\tau} \rho_{33}^{0}=-\frac{2|\mu|^{2}}{\hbar^{2}} v_{0} \int_{-\infty}^{\tau}\left(\sigma u_{0}+w_{2} v_{0}\right) .
$$

The $x$ and $y$ components $P_{0}$ and $Q_{0}$ of the zeroth-order polarization density $\vec{P}_{0}$ are given by

$$
\begin{aligned}
& P_{0}=N\left(\rho_{12}^{0} \mu^{*}+\rho_{12}^{0 *} \mu\right), \\
& Q_{0}=N\left(\rho_{13}^{0} \mu^{*}+\rho_{13}^{0 *} \mu\right) .
\end{aligned}
$$

By integrating (14) and (15), incorporating them into (22) and (23), and setting

$$
\kappa=\operatorname{Im}\left(\rho_{23}^{0}\right),
$$

we get

$$
\begin{gathered}
P_{0}=\frac{2|\mu|^{2} N}{\hbar} \int_{-\infty}^{\tau} \kappa v_{0}, \\
Q_{0}=-\frac{2|\mu|^{2} N}{\hbar} \int_{-\infty}^{\tau} \kappa u_{0} .
\end{gathered}
$$

By integrating Eqs. (14) and (15), incorporating them into (16), and separating real and imaginary parts, we obtain evolution equations for $\sigma=\operatorname{Re} \rho_{23}^{0}$ and $\kappa=\operatorname{Im} \rho_{23}^{0}$, as

$$
\partial_{\tau} \sigma=-u_{0} P_{x}-v_{0} P_{y},
$$

where we have set

$$
\begin{aligned}
& P_{x}=\frac{|\mu|^{2}}{\hbar^{2}} \int_{-\infty}^{\tau}\left(w_{1} u_{0}+\sigma v_{0}\right), \\
& P_{y}=\frac{|\mu|^{2}}{\hbar^{2}} \int_{-\infty}^{\tau}\left(w_{2} v_{0}+\sigma u_{0}\right),
\end{aligned}
$$

and

$$
\partial_{\tau} \kappa=\frac{-|\mu|^{2}}{\hbar^{2}}\left(u_{0} \int_{-\infty}^{\tau} \kappa u_{0}+v_{0} \int_{-\infty}^{\tau} \kappa v_{0}\right) .
$$

Since Eq. (30) is a linear homogeneous integro-differential equation, it is obviously satisfied by $\kappa=0$. Depending on the electric field component, it might admit a nonzero solution. Let us assume that such a solution exists, and call it $\kappa_{0}$. Then, $\kappa=\lambda \kappa_{0}$ also solves Eq. (30) for any real $\lambda$, and neither the boundary conditions $\lim _{\tau \longrightarrow-\infty} \kappa=0$ nor the value of the field allow us to determine $\lambda$. This shows that the nonzero solution would not have any physical meaning in the frame of the perturbation expansion, and forces us to consider the solution $\kappa=0$ only; consequently, $\rho_{23}^{0}=\sigma$ is real.

Then, assuming that it vanishes at infinity, it is found that

$$
\vec{P}_{0}=\overrightarrow{0} \text {. }
$$

By incorporating this value into the wave equation (5) at order $\varepsilon^{0}$, we get

$$
\frac{1}{V^{2}} \partial_{\tau}^{2} \vec{E}_{0}=\frac{1}{c^{2}} \partial_{\tau}^{2}\left(\vec{E}_{0}+4 \pi \vec{P}_{0}\right),
$$

which, taking (31) into account, is satisfied if the wave velocity is $V=c$ at the zeroth order of the series expansion in the small parameter $\varepsilon$.

\section{Order $\varepsilon^{1}$}

\section{Polarization density}

The Heisenberg equation (4) at order $\varepsilon^{1}$ is

$$
i \hbar \partial_{\tau} \rho^{1}=\left[H_{0}, \rho^{0}\right]-\left[\vec{\mu} \vec{E}_{0}, \rho^{1}\right]-\left[\vec{\mu} \vec{E}_{1}, \rho^{0}\right],
$$

and then we get

$$
\begin{aligned}
i \hbar \partial_{\tau} \rho_{11}^{1}= & -\left(\mu \rho_{12}^{1 *}-\rho_{12}^{1} \mu^{*}\right) u_{0}-\left(\mu \rho_{13}^{1 *}-\rho_{13}^{1} \mu^{*}\right) v_{0} \\
& -\left(\mu \rho_{12}^{0 *}-\rho_{12}^{0} \mu^{*}\right) u_{1}-\left(\mu \rho_{13}^{0 *}-\rho_{13}^{0} \mu^{*}\right) v_{1}, \\
i \hbar \partial_{\tau} \rho_{22}^{1}= & -\left(\mu^{*} \rho_{12}^{1}-\rho_{12}^{1 *} \mu\right) u_{0}-\left(\mu^{*} \rho_{12}^{0}-\rho_{12}^{0 *} \mu\right) u_{1}, \\
i \hbar \partial_{\tau} \rho_{33}^{1}= & -\left(\mu^{*} \rho_{13}^{1}-\rho_{13}^{1 *} \mu\right) v_{0}-\left(\mu^{*} \rho_{13}^{0}-\rho_{13}^{0 *} \mu\right) v_{1}, \\
i \hbar \partial_{\tau} \rho_{12}^{1}= & -\hbar \Omega \rho_{12}^{0}-\mu\left(\rho_{22}^{1}-\rho_{11}^{1}\right) u_{0}-\mu \rho_{23}^{1 *} v_{0} \\
& -\mu\left(\rho_{22}^{0}-\rho_{11}^{0}\right) u_{1}-\mu \rho_{23}^{0 *} v_{1}, \\
i \hbar \partial_{\tau} \rho_{13}^{1}= & -\hbar \Omega \rho_{13}^{0}-\mu \rho_{23}^{1} u_{0}-\mu\left(\rho_{33}^{1}-\rho_{11}^{1}\right) v_{0} \\
& -\mu \rho_{23}^{0} u_{1}-\mu\left(\rho_{33}^{0}-\rho_{11}^{0}\right) v_{1}, \\
i \hbar \partial_{\tau} \rho_{23}^{1}= & -\mu^{*} \rho_{13}^{1} u_{0}+\rho_{12}^{1 *} \mu v_{0}-\mu^{*} \rho_{13}^{0} u_{1}+\rho_{12}^{0 *} \mu v_{1} .
\end{aligned}
$$


The computation of the polarization density components $P_{1}$ and $Q_{1}$ involve the density matrix elements $\rho_{12}^{1}$ and $\rho_{13}^{1}$ respectively. The former is obtained from (37), in which $\rho_{12}^{0}$ is reported using (14) to yield

$$
\begin{aligned}
\partial_{\tau} \rho_{12}^{1}= & -\frac{\mu \Omega}{\hbar} \int_{-\infty}^{\tau}\left(w_{1} u_{0}+\sigma v_{0}\right)+\frac{i \mu}{\hbar}\left[\left(\rho_{22}^{1}-\rho_{11}^{1}\right) u_{0}\right. \\
& \left.+w_{1} u_{1}+\rho_{23}^{1 *} v_{0}+\sigma v_{1}\right] .
\end{aligned}
$$

Since

$$
P_{1}=N\left(\rho_{12}^{1} \mu^{*}+\rho_{12}^{1 *} \mu\right),
$$

the expression of $P_{1}$ becomes

$$
\begin{aligned}
P_{1}= & -\frac{2|\mu|^{2} \Omega N}{\hbar} \int_{-\infty}^{\tau} \int_{-\infty}^{\tau}\left(w_{1} u_{0}+\sigma v_{0}\right) \\
& +\frac{2|\mu|^{2} N}{\hbar} \int_{-\infty}^{\tau} \hat{\kappa} v_{0},
\end{aligned}
$$

in which we have set

$$
\hat{\kappa}=\operatorname{Im}\left(\rho_{23}^{1}\right) .
$$

In the same way, we have

$$
\begin{aligned}
\partial_{\tau} \rho_{13}^{1}= & -\frac{\mu \Omega}{\hbar} \int_{-\infty}^{\tau}\left(\sigma u_{0}+w_{2} v_{0}\right)+\frac{i \mu}{\hbar}\left[\left(\rho_{33}^{1}-\rho_{11}^{1}\right) v_{0}\right. \\
& \left.+w_{2} v_{1}+\rho_{23}^{1} u_{0}+\sigma u_{1}\right]
\end{aligned}
$$

and

$$
\begin{aligned}
Q_{1}= & -\frac{2|\mu|^{2} \Omega N}{\hbar} \int_{-\infty}^{\tau} \int_{-\infty}^{\tau}\left(\sigma u_{0}+w_{2} v_{0}\right) \\
& -\frac{2|\mu|^{2} N}{\hbar} \int_{-\infty}^{\tau} \hat{\kappa} u_{0} .
\end{aligned}
$$

\section{Equation for the electric field}

The wave equation (5), at order $\varepsilon^{1}$, yields

$$
\frac{1}{V^{2}} \partial_{\tau}^{2} \vec{E}_{1}-\frac{2}{V} \partial_{\tau} \partial_{\zeta} \vec{E}_{0}=\frac{1}{c^{2}} \partial_{\tau}^{2}\left(\vec{E}_{1}+4 \pi \vec{P}_{1}\right) .
$$

When we incorporate the expressions (42) and (45) of the components of $\vec{P}_{1}$ and take into account the fact that $V=c$, the terms involving $u_{1}$ and $v_{1}$ vanish from the equation, which reduces to

$$
\begin{aligned}
\partial_{\tau} \partial_{\zeta} u_{0}= & \frac{4 \pi|\mu|^{2} \Omega N}{\hbar c}\left(w_{1} u_{0}+\sigma v_{0}\right) \\
& -\frac{4|\mu|^{2} N}{\hbar c} \partial_{\tau}\left[\widehat{\kappa} v_{0}+\kappa v_{1}\right], \\
\partial_{\tau} \partial_{\zeta} v_{0}= & \frac{4 \pi|\mu|^{2} \Omega N}{\hbar c}\left(\sigma u_{0}+w_{2} v_{0}\right) \\
& +\frac{4|\mu|^{2} N}{\hbar c} \partial_{\tau}\left(\widehat{\kappa} u_{0}+\kappa u_{1}\right) .
\end{aligned}
$$

However, evolution equations for $w_{1}, w_{2}, \sigma$, and $\hat{\kappa}$ are required, and we derive them in what follows.

\section{Equations for $w_{1}, w_{2}$, and $\sigma$}

The evolution equations for the diagonal elements of $\rho^{0}$ are Eqs. (17), (20), and (21). The evolution equations for $w_{1}, w_{2}$ are deduced straightforwardly from them, as

$$
\begin{aligned}
& \partial_{\tau} w_{1}=-2 u_{0} P_{x}-v_{0} P_{y}, \\
& \partial_{\tau} w_{2}=-u_{0} P_{x}-2 v_{0} P_{y},
\end{aligned}
$$

where $P_{x}$ and $P_{y}$ are defined by (28) and (29) respectively. The evolution equation for $\sigma$ has already been computed in Eq. (27).

Then $\rho_{13}^{0}$ and $\rho_{12}^{0}$ are extracted from Eqs. (14) and (15) and incorporated into (39) to yield

$$
\begin{aligned}
\partial_{\tau} \rho_{23}^{1}= & \frac{i}{\hbar}\left(\mu^{*} \rho_{13}^{1} u_{0}-\rho_{12}^{1 *} \mu v_{0}\right)-\frac{|\mu|^{2}}{\hbar^{2}}\left[u_{1} \int_{-\infty}^{\tau}\left(\sigma u_{0}+w_{2} v_{0}\right)\right. \\
& \left.+v_{1} \int_{-\infty}^{\tau}\left(w_{1} u_{0}+\sigma v_{0}\right)\right] .
\end{aligned}
$$

Then we take the imaginary part of Eq. (51):

$$
\partial_{\tau} \hat{\kappa}=\frac{1}{\hbar}\left[\operatorname{Re}\left(\mu^{*} \rho_{13}^{1}\right) u_{0}-\operatorname{Re}\left(\mu^{*} \rho_{12}^{1}\right) v_{0}\right] .
$$

It follows from Eqs. (40) and (44) that

$$
\operatorname{Re}\left(\mu^{*} \rho_{12}^{1}\right)=-\hbar \Omega \int_{-\infty}^{\tau} P_{x},
$$

and

$$
\operatorname{Re}\left(\mu^{*} \rho_{13}^{1}\right)=-\hbar \Omega \int_{-\infty}^{\tau} P_{y},
$$

and consequently

$$
\partial_{\tau} \hat{\kappa}=-\Omega\left(u_{0} \int_{-\infty}^{\tau} P_{y}-v_{0} \int_{-\infty}^{\tau} P_{x}\right) .
$$

The set of coupled nonlinear equations (47), (48), (28), (29), (22), (23), (55), (49), (50), and (27) yields the sought nonlinear dynamical model, eventually. It contains two kinds of terms coupling the polarization components: One is the structure of the population inversion, which involves three components $w_{1}$, $w_{2}$, and $\sigma$, and the other one is the term involving $\hat{\kappa}$, which couples the components of the polarization density at the level of their dynamics. Only parts of these coupling terms were taken into account in previous studies [39,40]. Notice that the coupling terms are responsible for the instability of the $\mathrm{CP}$ pulse in the subcycle range.

\section{MATHEMATICAL PROPERTIES OF THE COUPLED SYSTEM OF NONLINEAR EQUATIONS}

We get the coupled system of equations:

$$
\begin{gathered}
\partial_{\zeta} u_{0}=\frac{-2 \pi}{c} \partial_{\tau} P_{1}, \\
\partial_{\zeta} v_{0}=\frac{-2 \pi}{c} \partial_{\tau} Q_{1}, \\
\partial_{\tau} P_{1}=-2 N \hbar \Omega P_{x}+\frac{2 N|\mu|^{2}}{\hbar} v_{0} \hat{\kappa}, \\
\partial_{\tau} Q_{1}=-2 N \hbar \Omega P_{y}-\frac{2 N|\mu|^{2}}{\hbar} u_{0} \hat{\kappa},
\end{gathered}
$$




$$
\begin{gathered}
\partial_{\tau} P_{x}=\frac{|\mu|^{2}}{\hbar^{2}}\left(w_{1} u_{0}+\sigma v_{0}\right), \\
\partial_{\tau} P_{y}=\frac{|\mu|^{2}}{\hbar^{2}}\left(w_{2} v_{0}+\sigma u_{0}\right), \\
\partial_{\tau} \hat{\kappa}=\frac{1}{2 N \hbar}\left(u_{0} Q_{1}-v_{0} p_{1}\right), \\
\partial_{\tau} w_{1}=-4 u_{0} P_{x}-2 v_{0} P_{y}, \\
\partial_{\tau} w_{2}=-2 u_{0} P_{x}-4 v_{0} P_{y}, \\
\partial_{\tau} \sigma=-\left(u_{0} P_{y}+v_{0} P_{x}\right) .
\end{gathered}
$$

\section{A. Normalization}

Setting

$$
(u, v)=\frac{1}{E_{0}}\left(u_{0}, v_{0}\right), \quad T=\frac{\tau}{T_{0}}, \quad Z=\frac{\zeta}{D},
$$

in which the reference electric field $E_{0}$, the reference propagation distance $D$, and the reference time $T_{0}$ are related through

$$
T_{0}=\frac{\hbar}{|\mu| E_{0}}, \quad D=\frac{E_{0} c}{4 \pi N \Omega|\mu|},
$$

and

$$
(m, n)=\frac{\hbar}{\mu}\left(P_{x}, P_{y}\right), \quad(p, q)=\frac{E_{0}}{2 N \hbar \Omega}\left(P_{1}, Q_{1}\right),
$$

system (56)-(65) reduces to

$$
\begin{gathered}
\partial_{Z} u=-\partial_{T} p, \\
\partial_{Z} v=-\partial_{T} q, \\
\partial_{T} p=-m+v K, \\
\partial_{T} q=-n-u K, \\
\partial_{T} m=w_{1} u+S v, \\
\partial_{T} n=w_{2} v+S u, \\
\partial_{T} K=u q-v p, \\
\partial_{T} w_{1}=-4 u m-2 v n, \\
\partial_{T} w_{2}=-2 u m-4 v n, \\
\partial_{T} S=-u n-v m,
\end{gathered}
$$

or, setting $w=\left(w_{1}+w_{2}\right) / 2, r=\left(w_{2}-w_{1}\right) / 2$, they reduce to

$$
\begin{gathered}
\partial_{Z} u=-\partial_{T} p, \\
\partial_{Z} v=-\partial_{T} q, \\
\partial_{T} p=-m+v K, \\
\partial_{T} q=-n-u K, \\
\partial_{T} m=(w-r) u+S v, \\
\partial_{T} n=(w+r) v+S u, \\
\partial_{T} K=u q-v p, \\
\partial_{T} w=-3(u m+v n), \\
\partial_{T} r=u m-v n, \\
\partial_{T} S=-u n-v m .
\end{gathered}
$$

Coming back from the scaled variables (with $\varepsilon$ ) to the original ones is simply performed by restoring $\Omega$ to its initial value. Then it is seen from (67) that the short-wave assumption mainly expresses in the fact that the reference propagation distance $D$ is large.

By setting

$$
P=p+i q, \quad M=m+i n, \quad U=u+i v, \quad s=r-i S,
$$

as new complex quantities, the coupled system of equations (79)-(88) can be written in a more compact form as

$$
\begin{gathered}
U_{Z}=-P_{T}, \\
P_{T}=-M-i U K, \\
M_{T}=w U-s U^{*}, \\
K_{T}=\operatorname{Im}\left(U^{*} P\right), \\
s_{T}=U M, \\
w_{T}=-3 \operatorname{Re}\left(U^{*} M\right) .
\end{gathered}
$$

\section{B. Conservation laws}

Let us denote by $I=u^{2}+v^{2}$ the normalized intensity. It is straightforward that

$$
I_{Z}=\frac{-2}{3} w_{T},
$$

which shows that when the homogeneous population inversion $w$ has its value corresponding to the thermal equilibrium both before and after the pulse, the power $\int_{-\infty}^{+\infty} I d t$ is conserved during propagation. It also shows that the energy transfer inside the pulse is entirely governed by the homogeneous population inversion $w$.

However, there is another conservation law. Let us consider the scalar case $v=0$. It is seen that $K, S, n, q$ are 0 and $w_{1}=2 w_{2}$. Then the system (79)-(88) reduces to

$$
\begin{gathered}
u_{Z}=-p_{T}, \\
p_{T}=-m, \\
m_{T}=w_{1} u, \\
w_{1 T}=-4 u m .
\end{gathered}
$$

System (97)-(100) reduces to the sine Gordon equation as follows (computation was given first in [43]; however, it is worth being detailed again here). To this aim, let us set

$$
m=A \sin \varphi, \quad w_{1}=2 A \cos \varphi .
$$

Then, direct computation shows $A_{T}=0$, and hence $A$ is a constant. From (97)-(99), it is seen that

$$
u_{Z}=A \sin \varphi
$$

On the other hand, $u_{Z T}$ can be computed, either by taking the $T$ derivative of (102), which yields $u_{Z T}=w_{1} \varphi_{T} / 2$, or by combining (97) and (99), which yields $u_{Z T}=w_{1} u$. Comparison between both expressions shows that $u=\varphi_{T} / 2$, and we get the $\mathrm{sG}$ equation

$$
\varphi_{Z T}=2 A \sin \varphi
$$


From this derivation, it is seen that the reduction of system (97)-(100) to $\mathrm{sG}$ is based on the conservation law

$$
\partial_{T} A^{2}=\partial_{T}\left(m^{2}+\frac{w_{1}^{2}}{4}\right)=0 .
$$

The question is whether (104) can be generalized to the vector case. The answer is yes. It is easy to show that the quantity

$$
\mathcal{A}=m^{2}+n^{2}+\frac{1}{3} w^{2}+r^{2}+S^{2}
$$

in terms of the real normalized variables, or

$$
\mathcal{A}=|M|^{2}+\frac{1}{3} w^{2}+|S|^{2}
$$

in terms of the complex ones, is conserved, which can be checked by direct computation of $\partial_{\tau} \mathcal{A}$. Let us notice, however, that the remaining of these transformations cannot be generalized to the vector system of nonlinear coupled equations.

\section{Linear polarizations}

We seek for solutions to system (69)-(78) of the form

$$
\left(\begin{array}{l}
u \\
v
\end{array}\right)=f\left(\begin{array}{c}
\cos \theta \\
\sin \theta
\end{array}\right),
$$

in which $\theta$ is the angle between the polarization direction and the $x$ axis. It is straightforward to see that

$$
\left(\begin{array}{l}
p \\
q
\end{array}\right)=\Phi\left(\begin{array}{c}
\cos \theta \\
\sin \theta
\end{array}\right)
$$

with

$$
\partial_{Z} f=-\partial_{T} \Phi,
$$

and hence $K=0$ from Eq. (75). Then Eqs. (71)-(72) yield

$$
\left(\begin{array}{c}
m \\
n
\end{array}\right)=R\left(\begin{array}{c}
\cos \theta \\
\sin \theta
\end{array}\right),
$$

with

$$
\partial_{T} \Phi=-R
$$

Equations (76)-(78) show that $w_{1}, w_{2}$, and $S$ must be proportional. Let us set $W$ so that

$$
\partial_{T} W=-4 f R
$$

and then we get

$$
\begin{aligned}
w_{1} & =\left(1+\cos \theta^{2}\right) \frac{W}{2}, \\
w_{2} & =\left(1+\sin \theta^{2}\right) \frac{W}{2}, \\
S & =\cos \theta \sin \theta \frac{W}{2} .
\end{aligned}
$$

Then the equation for $R$ is

$$
\partial_{T} R=W f
$$

and hence the amplitudes $f, \Phi, R$, and the reduced population inversion $W$ solve the scalar model (97)-(100) for any polarization angle $\theta$. Hence the vectorial model allows us to retrieve the linear polarization case.

\section{CIRCULAR POLARIZATION}

\section{A. Approximate expression of circularly polarized pulses}

A circularly polarized FCP is more easily described by means of the complex system (90)-(95). It would correspond to a solution of the form

$$
U=F\left(T-\frac{Z}{v_{g}}\right) e^{i(k Z-\omega T)} .
$$

Direct substitution of (117) into the system (90)-(95) shows (after some computation, details of which are omitted here) that no exact solution of this form exist. The nonexistence of exact non-SVEA circularly polarized pulse solutions to the Maxwell-Bloch equations was already pointed out in [39], although in the frame of a slightly different model.

We can seek for approximate solutions in the limit of large $\omega$ (i.e., in the SVEA limit) by means of a multiscale expansion close to the standard one for deriving a NLS model in the SVEA limit [44]. We consider some small parameter $\varepsilon$, so that $1 / \varepsilon$ is the order of magnitude of the number of optical cycles in the pulse, assumed to be large. We expand

$$
U=\sum_{n \geqslant 1, r} \varepsilon^{n} e^{i r \varphi} U_{r, n}
$$

with $\varphi=k Z-\omega T$, and introduce slow variables

$$
\tau=\varepsilon\left(t-\frac{z}{v_{g}}\right), \quad \zeta=\varepsilon^{2} Z .
$$

At leading order, we assume that $U_{1,1}=F$ is the only nonzero term. In contrast with the standard derivation, $U_{-1,1}=0$ instead of $F^{*}$. The other variables $P, M, K, s$, and $w$ are expanded in the same way as $U$ is, except that we allow a zeroth-order term for $w$ and $s$.

The expansion is incorporated into system (90)-(95) and solved order by order. At leading order $\epsilon$, we get

$$
P_{1,1}=\frac{k}{\omega} F, \quad M_{1,1}=i k f,
$$

the other $P_{r, 1}$ and $M_{r, 1}$ being zero. The dispersion relation is found as

$$
w_{0,0}=\omega k,
$$

and $s_{0,0}=0$. It is also seen that $K_{r, 1}=s_{r, 1}=w_{r, 1}=0$ for any nonzero $r$.

At order $\varepsilon^{2}$, we obtain the velocity $v_{g}=-\omega / k$,

$$
\begin{gathered}
P_{1,2}=\frac{2 k}{i \omega^{2}} \partial_{\tau} F+\frac{k}{\omega} U_{1,2}, \\
M_{1,2}=i k U_{1,2}+\frac{k}{\omega} \partial_{\tau} F-i K_{0,1} F,
\end{gathered}
$$

and

$$
P_{r, 2}=\frac{k}{\omega} U_{r, 2}, \quad M_{r, 2}=\operatorname{irk} U_{r, 2},
$$

for all nonzero $r$. It is also seen that $w_{0,1}=-\omega K_{0,1}, s_{0,1}=0$, $U_{r, 2}=0$ for all $r \neq 1$, while $U_{1,2}$ is free. A nonlinear terms is computed:

$$
s_{2,2}=\frac{-k}{2 \omega} F^{2},
$$

while $s_{r, 2}=0$ for $r \neq 0,2$ and $K_{r, 2}=0$ for $r \neq 0$. 
Other relevant nonlinear terms are sought at order $\varepsilon^{3}$ :

$$
K_{0,2}=\frac{-k}{\omega^{2}}|F|^{2}, \quad w_{0,2}=\frac{-3 k}{2 \omega}|F|^{2} .
$$

The equation for the fundamental Fourier component $r=1$ of $U$ at order $\varepsilon^{3}$, after cancellation of the terms involving $U_{1,2}$ and $U_{1,3}$ and substitution of the various terms computed above (especially the nonlinear ones), yields the following nonlinear evolution equation:

$$
\partial_{\zeta} F=\frac{-i k}{\omega^{2}} \partial_{\tau}^{2} F-K_{0,1} \partial_{\tau} F-\frac{2 i k}{\omega^{2}} F|F|^{2},
$$

which is the NLS equation. The term involving $K_{0,1}$ represents only a shift in the velocity; its physical meaning is not clear. We can restrict ourselves to the case when $w_{0,1}=K_{0,1}=0$.

Let $F$ be the fundamental soliton solution to the NLS equation (127),

$$
F=\beta e^{\frac{-i \beta^{2} k}{\omega^{2}} \zeta} \operatorname{sech} \beta \tau
$$

It accounts for a circularly polarized FCP. The corresponding approximate expression of $U$ is

$$
U=b e^{i\left[\left(k-\frac{b^{2} k}{\omega^{2}}\right) Z-\omega T\right]} \operatorname{sech}\left[b\left(T+\frac{k}{\omega} Z\right)\right],
$$

in which the soliton parameter $b$ is $b=\varepsilon \beta$ and assumed to be small.

The nonzero nonlinear terms which are involved in the evolution of the circularly polarized FCP (129) are $s_{2,2}, w_{0,2}$, and $K_{0,2}$, i.e., the second harmonic for the cross term of population inversion $s$, the rectified term of the population inversion $w$, and the coupling polarization term $K$.

\section{B. Stability of the circularly polarized soliton in the SVEA}

\section{Derivation of a system of two coupled NLS equations}

The stability of the circularly polarized pulse (129) can be addressed analytically within the SVEA. Therefore, we first compute the slowly varying envelope approximation of the system (69)-(78). The real form of the system is required, since each real component will be expanded in both a Fourier series and a series of power of $\varepsilon$, as

$$
u=\sum_{n \geqslant 1, r} \varepsilon^{n} e^{i r \varphi} u_{r, n},
$$

with $u_{1,1}=f, u_{-1,1}=f^{*}$, and so on, and respectively, $v_{1,1}=$ $g$. We use the same phase $\varphi$ and the same scaled variables $\zeta$, $\tau$ as in previous section [Eq. (119)].

At order $\varepsilon^{1}$ we get

$p_{1,1}=\frac{k}{\omega} f, \quad q_{1,1}=\frac{k}{\omega} g, \quad m_{1,1}=i k f, \quad n_{1,1}=i k g$,

the other components of $p, q, m, n, K$ at order 1 being zero, and $f$ and $g$ remain free if

$$
S_{0,0}=0, \quad w_{1,0,0}=w_{2,0,0}=\omega k,
$$

the other components of $w_{1}, w_{2}, S$ at order 0 being zero.
At order $\varepsilon^{2}$ we get

$$
\begin{gathered}
p_{1,2}=\frac{k}{\omega} u_{1,2}+\frac{i}{\omega}\left(\frac{1}{v_{g}}-\frac{k}{\omega}\right) \partial_{\tau} f, \\
q_{1,2}=\frac{k}{\omega} v_{1,2}+\frac{i}{\omega}\left(\frac{1}{v_{g}}-\frac{k}{\omega}\right) \partial_{\tau} g, \\
m_{1,2}=i k u_{1,2}-\frac{1}{v_{g}} \partial_{\tau} f+K_{0,1} g, \\
n_{1,2}=i k v_{1,2}-\frac{1}{v_{g}} \partial_{\tau} g-K_{0,1} f, \\
p_{r, 2}=\frac{k}{\omega} u_{r, 2}, \quad q_{r, 2}=\frac{k}{\omega} v_{r, 2}, \\
m_{r, 2}=i r k u_{r, 2}, \quad n_{r, 2}=i r k v_{r, 2} .
\end{gathered}
$$

The solvability condition of the equations for $u$ and $v$, for $r=1$, at this order, yield $v_{g}=-\omega / k$ as in previous section.

Next we get the nonlinear terms

$$
\begin{gathered}
w_{1,2,2}=\frac{k}{\omega}\left(2 f^{2}+g^{2}\right), \\
w_{2,2,2}=\frac{k}{\omega}\left(f^{2}+2 g^{2}\right), \\
S_{2,2}=\frac{k}{\omega} f g,
\end{gathered}
$$

and their complex conjugate $(r=-2)$, while the components with $r=0$ remain free, and the other components are zero, including $K_{2,2}$.

The nonlinear rectified terms are found at order $\varepsilon^{3}$, and they are

$$
\begin{aligned}
K_{0,2} & =\frac{2 i k}{\omega^{2}}\left(f g^{*}-f^{*} g\right), \\
w_{1,0,2} & =\frac{-k}{\omega}\left(4|f|^{2}+2|g|^{2}\right), \\
w_{2,0,2} & =\frac{-k}{\omega}\left(2|f|^{2}+4|g|^{2}\right), \\
S_{0,2} & =\frac{-k}{\omega}\left(f g^{*}+f^{*} g\right) .
\end{aligned}
$$

As in the previous section, the equations for the fundamental Fourier component $r=1$ of $u$ and $v$, at order $\varepsilon^{3}$, after cancellation of the terms involving $u_{1,2}, v_{1,2}, u_{1,3}$, and $v_{1,3}$ and substitution of the various terms computed above (especially the nonlinear ones), yields a system of two coupled nonlinear equations:

$$
\begin{gathered}
\partial_{\zeta} f=\frac{-i k}{\omega^{2}} \partial_{\tau}^{2} f-\frac{2 i k}{\omega^{2}}\left(f|f|^{2}+2 f|g|^{2}-f^{*} g^{2}\right), \\
\partial_{\zeta} g=\frac{-i k}{\omega^{2}} \partial_{\tau}^{2} g-\frac{2 i k}{\omega^{2}}\left(g|g|^{2}+2 g|f|^{2}-g^{*} f^{2}\right) .
\end{gathered}
$$

The system yielded by Eqs. (146) and (147) is a system of two coupled NLS equations, presenting both self- and crossphase-modulation, and four-wave mixing terms.

Before we proceed to the stability analysis of the circularly polarized pulse (117), let us consider more generally the solutions of (146) and (147) corresponding to a fixed polarization. If the polarization is fixed, we have $g=d f$ with $d$ some 
constant. By incorporating $g=d f$ into Eqs. (146) and (147), we obtained two different equations, which coincide if

$$
|d|^{2}-d^{2}=1-\frac{d^{*}}{d}
$$

Equation (148) is solved by separating amplitude and phase of $d$, and the solutions are $d$ real, or $d= \pm i$. Hence the "eigenpolarizations" of the system yielded by (146) and (147) are the linear polarizations and the two circular ones. For a circular polarization, Eqs. (146) and (147) reduce to

$$
\partial_{\zeta} f=\frac{-i k}{\omega^{2}} \partial_{\tau}^{2} f-\frac{8 i k}{\omega^{2}} f|f|^{2} .
$$

It is seen that the cross-phase and four-wave mixing terms add up in this case, while they cancel each other in the case of linearly polarized pulses.

The complex field is $U=u+i v$, with $u \simeq \varepsilon\left(f e^{i \varphi}+\right.$ $\left.f^{*} e^{-i \varphi}\right)$ and $v \simeq \varepsilon\left(g e^{i \varphi}+g^{*} e^{-i \varphi}\right)$, hence for $f=i g, U \simeq$ $\varepsilon 2 i g e^{i \varphi}$, and the amplitude considered in previous section is $F=2 f$, which proves that (149) coincides with (127).

\section{Stability}

Let us change $\zeta$ into $z=\frac{-2 k}{\omega^{2}} \zeta$. Equations (146) and (147) become

$$
\begin{gathered}
i \partial_{z} f+\partial_{\tau}^{2} f+f|f|^{2}+2 f|g|^{2}-f^{*} g^{2}=0, \\
i \partial_{z} g+\partial_{\tau}^{2} g+g|g|^{2}+2 g|f|^{2}-g^{*} f^{2}=0 .
\end{gathered}
$$

In the same way, the equation for the circular polarization becomes

$$
i \partial_{\zeta} g+\partial_{\tau}^{2} g+4 g|g|^{2}=0 .
$$

Let $g_{0}$ be the fundamental soliton solution to Eq. (152), so that the corresponding $U$ is the circularly polarized pulse (129).

Consider a perturbation of this pulse, defined by

$$
\begin{gathered}
f=i g_{0}+\eta f_{1}, \\
g=g_{0}+\eta g_{1},
\end{gathered}
$$

where $\eta$ is a small parameter. Equations (153) and (154) are incorporated into Eqs. (150) and (151), and the following evolution equations for $f_{1}$ and $g_{1}$ are derived at first order in $\eta$ :

$$
\begin{gathered}
i \partial_{z} f_{1}+\partial_{\tau}^{2} f_{1}+4 f_{1}\left|g_{0}\right|^{2}-2 f_{1}^{*} g_{0}^{2} \\
+4 i g_{1}\left|g_{0}\right|^{2}+2 i g_{1}^{*} g_{0}^{2}=0, \\
i \partial_{z} g_{1}+\partial_{\tau}^{2} g_{1}+4 g_{1}\left|g_{0}\right|^{2}+2 g_{1}^{*} g_{0}^{2} \\
-4 i f_{1}\left|g_{0}\right|^{2}+2 i f_{1}^{*} g_{0}^{2}=0 .
\end{gathered}
$$

Then, setting

$$
\psi_{ \pm}=\frac{1}{2} g_{1} \pm \frac{i}{2} f_{1}
$$

we get

$$
\begin{gathered}
i \partial_{z} \psi_{-}+\partial_{\tau}^{2} \psi_{-}+8 \psi_{-}\left|g_{0}\right|^{2}+4 g_{0}^{2} \psi_{-}^{*}=0, \\
i \partial_{z} \psi_{+}+\partial_{\tau}^{2} \psi_{+}=0 .
\end{gathered}
$$

Equation (158) is nothing else but the NLS equation (152) linearized about its solution $g_{0}$. From the IST it is well known that $g_{0}$ is a stable solution of Eq. (152), and hence

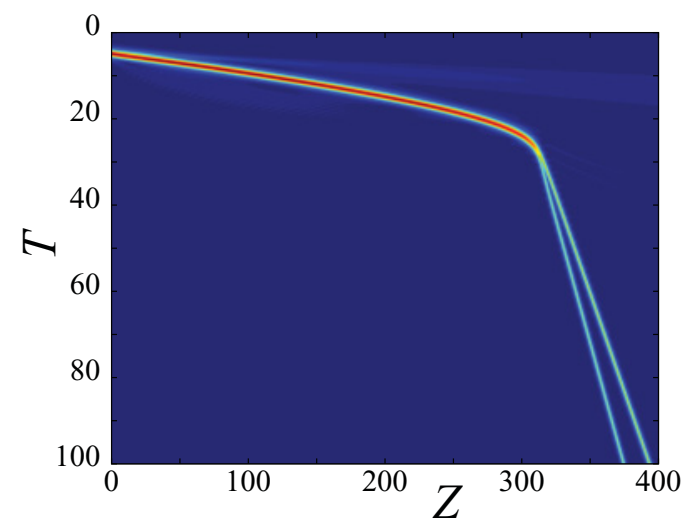

FIG. 1. (Color online) The circularly polarized FCP and its decay into orthogonally polarized single-humped pulse. Evolution in the $(Z, T)$ plane. Parameters are $\omega=5, b=2$. Here $Z_{\text {disp }}=37$.

no instability can arise in $\psi_{-}$. Equation (159) is easily solved by seeking solutions of the form $\psi_{+}=\exp i\left(k^{\prime} z-\omega^{\prime} \tau\right)$; the dispersion relation is $k^{\prime}=-\omega^{\prime 2}$. Since $k^{\prime}$ is real for any real $\omega$, no instability occurs from $\psi_{+}$. Consequently the circularly polarized pulse is stable.

\section{LIFETIME OF CIRCULARLY POLARIZED FEW-CYCLE PULSES AND TRANSITION TO SINGLE-HUMPED PULSES}

The existence and stability of the circularly polarized pulse within SVEA do not ensure either its stability or even its existence beyond SVEA [39]. In the following, we investigate numerically the stability of circularly polarized few-cycle pulses beyond SVEA. System (79)-(88) is solved numerically in the domain $Z \geqslant 0, T \geqslant 0$ as follows. The $Z$ evolution of $u$ and $v$ is computed by means of a standard fourth-order Runge-Kutta algorithm. At each step and substep of the scheme, the eight other components are computed using the same algorithm but relative to the $T$ variable. It involves values of $u$ and $v$ at intermediary times, which are computed by means of a polynomial interpolation over four existing points. The procedure does not allow speed compensation or periodic boundary conditions, and hence the propagation distance is limited by the size of the numerical box in $T$.

We assume that all atoms are initially in the fundamental state: $w=-1$ at $T=0$. We use the approximate circularly polarized pulse (129) as an input, with $\omega=5$, and vary the pulse duration $b$. An instability occurs: The FCP decays into linearly polarized single-humped pulses. In general, two orthogonally polarized pulses with different amplitudes are obtained (see Figs. 1, 2, and 3). For the shortest subcycle pulses, the instability occurs very fast, the amplitudes of the two single humped pulses strongly differ, and the angle between their polarization directions is not close to $\pi / 2$. The single-humped pulses are fundamental solitons of the $\mathrm{sG}$ equation (103) to which the system reduces in the case of linear polarizations.

The distance at which the transition occurs was also evaluated. In fact, the instability process has some duration. First, the amplitude starts to decay, then the internal oscillations slow 


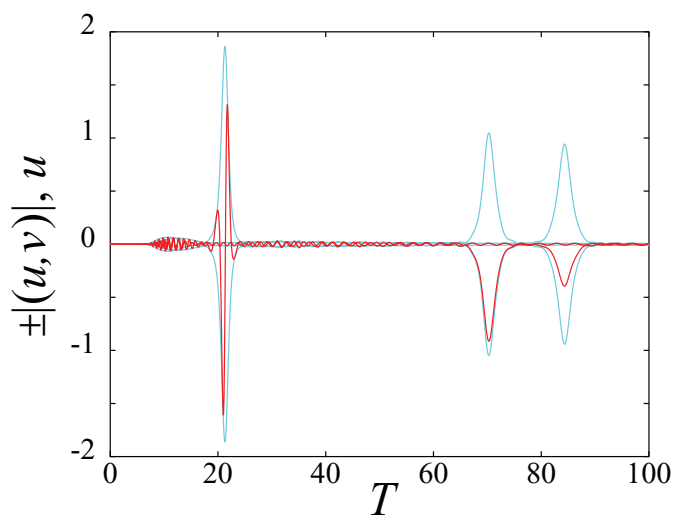

FIG. 2. (Color online) The circularly polarized FCP and its decay into orthogonally polarized single-humped pulse (same as Fig. 1). The shape of the FCP is shown at $Z=280$ (left) and $Z=360$ (right). Light blue (gray) denotes the amplitude $|U|=\sqrt{u^{2}+v^{2}}$ (and $-|U|$ ), and red (black) denotes $u=\operatorname{Re}(U)$.

down, and finally the pulse splits. We measure the distances $Z_{i}$ and $Z_{f}$ of the beginning and start of the process and take their mean value $Z_{\max }$. It is then compared to the dispersion length $Z_{\text {disp. }}$. The latter is computed as follows: We use the same code and the same initial data but strongly decrease the amplitude to compute the linear dispersion. Then the pulse duration [at full width at half maximum (FWHM)] is computed for each $Z$. It evolves linearly; the parameters of this evolution are computed using a least squares algorithm, from which the dispersion length $Z_{\text {disp }}$ is computed. $Z_{\text {disp }}$ is defined as the length at which the pulse length is twice its initial value. It is seen that $Z_{\max } / Z_{\text {disp }}$ evolves exponentially with respect to the pulse length (Fig. 4). The pulse duration may be measured by the ratio $\omega / b$; however, we can also consider the number of optical cycles $N_{c}$, which is the ratio of the pulse duration (FWHM $=2 \ln [1+\sqrt{(2)] / b}$ ) divided by the optical period $2 \pi / \omega$. The curve $Z_{\max } / Z_{\text {disp }}$ versus $N_{c}$ is shown in

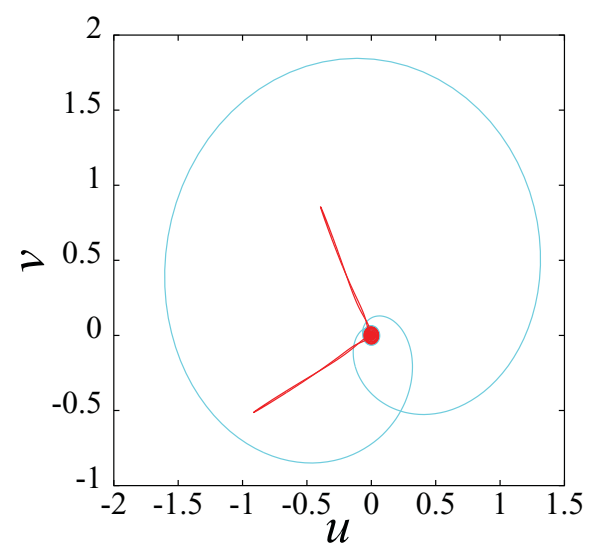

FIG. 3. (Color online) The circularly polarized FCP and its decay into orthogonally polarized single-humped pulse (same as Fig. 1). The trajectories of the tip of the normalized electric field vector $(u, v)$ are in the transverse plane, for the two same values of $Z$, showing the polarization. Light blue (gray) denotes $Z=280$ (left), and red (black) denotes $Z=360$.

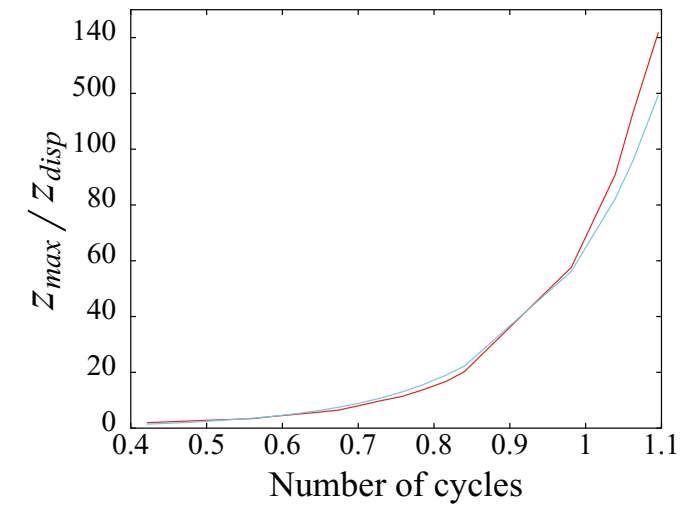

FIG. 4. (Color online) Plot of the lifetime of the circularly polarized FCP, measured in units of the dispersion length, against the number of optical cycles in the pulse. The red (black) line shows the numerical data. The light blue (gray) line shows the exponential fit.

Fig. 4, together with an exponential fit obtained by linear least squares in semilogarithmic representation. Properly speaking, no stability threshold for the circularly polarized FCPs can be evidenced; however, the lifetime becomes very long for $N_{c}>1$ (see Fig. 4). Notice that in Ref. [40], only the coupling through a common single population inversion (homogeneous broadening) was considered. This allowed the derivation of a non-SVEA pulse solution [40]. The frequency modulation of this non-SVEA pulse solution becomes as large as the frequency itself for $\omega / b=1 / 2 \sqrt{3}$, that is, for a number of cycles $N_{c} \simeq 0.08$. The result obtained in Ref. [40] presents some analogy with the transition from a circularly polarized oscillating pulse to the single-humped linearly polarized one that we described here, but would be smooth and occur at $N_{c} \simeq 0.08$ instead of $N_{c} \simeq 1$. We stress that the instability of the circularly polarized FCPs for subcycle pulses is thus strongly related to the coupling between polarization components.

\section{CONCLUSIONS}

The powerful multiscale perturbation analysis was used to derive evolution equations governing the propagation of circularly polarized femtosecond optical solitons in optical media beyond the slowly varying envelope approximation and in the short-wave-approximation regime. We took into account the vectorial character of the electric field, and therefore we properly considered the wave polarization effects. We have found that the two interacting wave forms corresponding to such vector few-optical-cycle solitons are adequately described by a coupled system of nonlinear equations at the first-order approximation of the perturbation approach. By direct numerical simulations, we calculated the lifetime of circularly polarized few-optical-cycle solitons, and we studied their decay into two orthogonally polarized single-humped pulses as a generic route of their instability. A challenging extension suggested by the present work is to consider the case of two optical transitions, one below and one above the range of propagated wavelengths. Another interesting issue is the generalization of the present study to one or even 
to two spatial transverse dimensions, in addition to time and spatial longitudinal coordinates, that is, to investigate vector few-optical-cycle spatiotemporal solitons (ultrashort circularly polarized light bullets) beyond the slowly varying envelope approximation.

\section{ACKNOWLEDGMENTS}

The work of D.M. at Laboratoire de Photonique d'Angers has been supported by the Région Pays de Loire, France.
[1] M. Wegener, Extreme Nonlinear Optics (Springer, Berlin, 2005).

[2] E. Goulielmakis et al., Science 320, 1614 (2008).

[3] A. Scrinzi, M. Yu. Ivanov, R. Kienberger, and D. M. Villeneuve, J. Phys. B 39, R1 (2006).

[4] T. Brabek and F. Krausz, Rev. Mod. Phys. 72, 545 (2000).

[5] G. Krauss, S. Lohss, T. Hanke, A. Sell, S. Eggert, R. Huber, and A. Leitenstorfer, Nat. Photonics 4, 33 (2010).

[6] X. Tan, X. Fan, Y. Yang, and D. Tong, J. Mod. Opt. 55, 2439 (2008).

[7] N. N. Rosanov, V. E. Semenov, and N. V. Vyssotina, Laser Phys. 17, 1311 (2007); Quantum Electron. 38, 137 (2008).

[8] A. Nazarkin, Phys. Rev. Lett. 97, 163904 (2006).

[9] A. I. Maimistov, Quantum Electron. 40, 756 (2010).

[10] T. Brabec and F. Krausz, Phys. Rev. Lett. 78, 3282 (1997).

[11] M. V. Tognetti and H. M. Crespo, J. Opt. Soc. Am. B 24, 1410 (2007).

[12] A. A. Voronin and A. M. Zheltikov, Phys. Rev. A 78, 063834 (2008).

[13] A. Kumar and V. Mishra, Phys. Rev. A 79, 063807 (2009).

[14] N. N. Akhmediev, I. V. Mel'nikov, and A. V. Nazarkin, Sov. Phys. Lebedev. Inst. Rep. 2, 66 (1989) [Kratk. Soobshch. Fiz. FIAN 2, 49 (1989)]; E. M. Belenov and A. V. Nazarkin, JETP Lett. 51, 288 (1990) [Pis'ma Zh. Eksp. Teor. Fiz. 51, 252 (1990)]; A. I. Maimistov and S. O. Elytin, J. Mod. Opt. 39, 2201 (1992); A. E. Kaplan and P. L. Shkolnikov, Phys. Rev. Lett. 75, 2316 (1995); S. V. Sazonov, JETP 92, 361 (2001).

[15] I. V. Mel'nikov, D. Mihalache, F. Moldoveanu, and N.-C. Panoiu, Phys. Rev. A 56, 1569 (1997); JETP Lett. 65, 393 (1997).

[16] H. Leblond and F. Sanchez, Phys. Rev. A 67, 013804 (2003).

[17] I. V. Mel'nikov, H. Leblond, F. Sanchez, and D. Mihalache, IEEE J. Sel. Top. Quantum Electron. 10, 870 (2004).

[18] H. Leblond, S. V. Sazonov, I. V. Mel'nikov, D. Mihalache, and F. Sanchez, Phys. Rev. A 74, 063815 (2006).

[19] H. Leblond, I. V. Mel'nikov, and D. Mihalache, Phys. Rev. A 78, 043802 (2008); H. Leblond and D. Mihalache, ibid. 79, 063835 (2009); J. Optoelectronics Adv. Mat. 12, 1 (2010).

[20] S. A. Skobelev, D. V. Kartashov, and A. V. Kim, Phys. Rev. Lett. 99, 203902 (2007).

[21] Sh. Amiranashvili, A. G. Vladimirov, and U. Bandelow, Phys. Rev. A 77, 063821 (2008).
[22] L. Bergé and S. Skupin, Phys. Rev. Lett. 100, 113902 (2008).

[23] H. Leblond, D. Kremer, and D. Mihalache, Phys. Rev. A 80, 053812 (2009).

[24] H. Leblond, D. Kremer, and D. Mihalache, Phys. Rev. A 81, 033824 (2010); H. Leblond and D. Mihalache, ibid. 81, 063815 (2010).

[25] X.-T. Xie and M. A. Macovei, Phys. Rev. Lett. 104, 073902 (2010).

[26] P. Dombi et al., Opt. Express 18, 24206 (2010).

[27] N. N. Rosanov, V. V. Kozlov, and S. Wabnitz, Phys. Rev. A 81, 043815 (2010).

[28] H. Leblond and D. Mihalache, J. Phys. A 43, 375205 (2010).

[29] E. D. Farnum and J. Nathan Kutz, Opt. Lett. 35, 3033 (2010).

[30] Q. Lin, J. Zheng, J. Dai, I. Chen Ho, and X.-C. Zhang, Phys. Rev. A 81, 043821 (2010).

[31] X. Song, W. Yang, Z. Zeng, R. Li, and Z. Xu, Phys. Rev. A 82, 053821 (2010).

[32] R. K. Dodd, J. C. Eilbeck, J. D. Gibbon, and H. C. Morris, Solitons and Nonlinear Wave Equations (Academic Press, London, 1982).

[33] M. J. Ablowitz and H. Segur, Solitons and the Inverse Scattering Transform (SIAM, Philadelphia, 1981).

[34] K. Konno, W. Kameyama, and H. Sanuki, J. Phys. Soc. Jpn. 37, 171 (1974).

[35] M. Pietrzyk, I. Kanattšikov, and U. Bandelow, J. Nonlin. Math. Phys. 15, 162 (2008).

[36] V. G. Bespalov, S. A. Kozlov, Yu. A. Shpolyansky, and I. A. Walmsley, Phys. Rev. A 66, 013811 (2002).

[37] H. Leblond, H. Triki, F. Sanchez, and D. Mihalache, Phys. Rev. A 83, 063802 (2011).

[38] L. Allen and J. H. Eberly, Optical Resonance and Two-Level Atoms (Dover, New York, 1987).

[39] S. V. Branis, O. Martin, and J. L. Birman, Phys. Rev. A 43, 1549 (1991).

[40] A. V. Andreev, JETP 81, 434 (1995) [Zh. Eksp. Teor. Fiz. 108, 796 (1995)].

[41] M. A. Manna and V. Merle, Phys. Rev. E 57, 6206 (1998).

[42] M. A. Manna, J. Phys. A 34, 4475 (2001).

[43] R. A. Kraenkel, M. A. Manna, and V. Merle, Phys. Rev. E 61, 976 (2000).

[44] H. Leblond, J. Phys. B 41, 043001 (2008). 